\title{
Applicability of ANN models and Taguchi method for the determination of tool life in turning
}

\author{
Nikolaos E. Karkalos, and Angelos P. Markopoulos* \\ ${ }^{1}$ National Technical University of Athens, School of Mechanical Engineering, Section of \\ Manufacturing Technology, Heroon Polytechniou 9, 15780, Athens, Greece
}

\begin{abstract}
Tool life is an important parameter in machining processes, affecting directly the quality of machined components and the process cost. It is already shown that various parameters can affect tool life such as process parameters, i.e. depth of cut, cutting speed and feed, or material properties of cutting tool and workpiece. The determination of the effect of each parameter on tool life is of crucial importance when designing the manufacturing process of a product in order to select suitable process parameter values and tool types. Several empirical formulas for the determination of tool life exist in the relevant literature; especially in the case of CBN cutting tools for turning, a cubic polynomial formula was proposed to model the relationship between tool life and cutting speed. The determination of the polynomial parameters was performed by conducting cutting experiments for several cutting speeds, without the aid of a design of experiments (DoE) method in order to model properly this non-linear relationship. In this paper, the feasibility of determining this non-linear relationship by conducting experiments designed by Taguchi method and using artificial neural networks (ANN) is investigated for several cases and conclusions on the applicability of this approach are presented.
\end{abstract}

\section{Introduction}

The determination of the correlation between tool life (and tool wear) and various process parameters is essential for efficient manufacturing. Tool wear refers to the gradual deterioration of cutting tools' performance due to various factors, e.g. thermo-mechanical or chemical ones. Some of the types of tool wear include flank wear, crater wear and notch wear among others; their effects on the process, apart from increased cutting forces and temperature are the decreased dimensional accuracy and quality of produced parts and tool breakage, which eventually result in increased cost.

As far as tool wear models are concerned, apart from the empirical or semi-empirical approaches [1-2], firstly established from the early 1900s, there also exist some numerical

\footnotetext{
* Corresponding author: amark@mail.ntua.gr
} 
and several soft computing models, capable of predicting cutting tool wear in various cases. Leone et al. [3] employed an ANN model for the prediction of tool wear progression during machining of a Nickel-based alloy. They found that it was superior from a regression model, as it was able to predict maximum flank wear $\left(V B_{\max }\right)$ for cutting speeds more than $50 \mathrm{~m} / \mathrm{min}$, which was the safe machining limit for the examined cases, with significant accuracy. Rao et al. [4] used an ANN model for the prediction of tool wear, surface roughness and vibration of workpiece during boring. They conducted experiments designed by Taguchi method with two levels of cutting speed, nose radius and feed values. The proposed model consisted of four layers and exhibited mean percentage error of $4.52 \%$, $4.26 \%$ and $2.92 \%$ for surface roughness, amplitude of vibration and tool wear, respectively.

D'Addona et al. [5] developed an ANN model of tool wear during machining of nickel superalloys. For the model, data from experiments at five different cutting speeds were employed and the ANN contained three layers; hidden layer neurons number was determined by a "cascade learning" procedure in which new nodes are added to the model until no further reduction of error is observed. Root mean square error (RMSE) of the results up to the largest cutting speed value were acceptable, whereas an underestimation of the wear land size was observed for the cutting speed of $55 \mathrm{~m} / \mathrm{min}$ when the wear value was over the limit value. D'Addona and Teti [6] created a predictive tool for crater wear using image data of the cutting tool and an ANN model. They conducted tests for different values of cutting speed, feed rate and depth of cut. After images corresponding to each studied cases were properly processed, a three-layer ANN was used to predict tool wear and comments were made on the correct strategies for tool wear recognition.

However, ANN models are often employed for on-line monitoring of machining processes as well. Srikant et al. [7] used a modified back propagation ANN for online tool wear prediction in cases of wet machining. They conducted various experiments with dry and wet conditions and various cutting fluids and obtained results concerning cutting forces, temperature, flank wear and surface roughness. The inputs for the ANN model were forces, temperature, surface roughness, machining time, viscosity and thermal conductivity of cutting fluid and workpiece hardness and the structure of neural network was optimized using simulated annealing method. Absolute error of prediction was lower than 5\%, thus the results were considered satisfying. Corne et al. [8] created a real time tool wear and breakage prediction system using ANN. They conducted experiments of hole drilling and observed tool wear during the process. For the development of the ANN model, they used data from five different experiments with spindle speed between 400 and $600 \mathrm{rpm}$ and feed of 0.05 and $0.1 \mathrm{~mm} / \mathrm{rev}$, for a total of 36 input/output data samples. Levenberg-Marquardt training algorithm and a single hidden layer of five neurons were chosen. They compared the performance of models with direct power and force measurement and observed that models with force input data were more accurate, but given power and force are correlated, measurement of power load during the process can lead to reliable prediction of tool wear.

Liu and Altintas [9] employed an ANN for the online monitoring of flank wear. The input variables of the model were cutting speed, feed rate, and cutting force ratio as well as past values of measured force ratio and estimated flank wear and the data covered ten different cutting conditions. The real time monitoring system was implemented and the performance of tool wear prediction model was found to be in significant agreement with test results on new turning experiments. Krishnakumar et al. [10] developed a tool wear predictive tool based on vibration signals monitoring for high speed machining of Ti alloy. They conducted six machining experiments with various cutting speed and spindle speed values for a total of 87 data samples and created an ANN model with standard error, kurtosis and median or vibration data as inputs. It was found that the ANN model was superior from a decision tree model for the classification of cutting tool condition. Other 
notable studies with neural network models include studies with neuro-fuzzy approaches $[11,12]$.

In the current work, the applicability of standard DoE methods and ANN models for the approximation of tool life-cutting speed relationship is investigated. The tool life curves derived from the ANN models are directly compared to the experimental ones in order to determine up to which degree tool life and tool life curve characteristics, such as minima and maxima, can be approximated.

\section{Tool life models}

Tool life models are often developed in respect to process parameters. Some of the most notable tool life equations include Taylor, Safonov, Wu and Granovski among others [2]. The simplest among them is the well-known Taylor equation for tool life which correlates cutting speed $\left(v_{c}\right)$ with tool life $(T)$ and tool-workpiece characteristics and can provide a preliminary approximation for tool life:

$$
v_{c} T^{n}=c
$$

where $n$ and $c$ are constants depending on cutting tool and workpiece. This equation can be generalized to include depth of cut $\left(a_{p}\right)$ and feed rate $(f)$ as well:

$$
v_{c} T^{n} a_{p}^{x} f^{y}=c
$$

where $n, x, y, c$ are constants, which are experimentally derived. Moreover, some approaches consider other process-related parameters and even the value of $V B_{\max }$. However, for the case of machining with CBN tools, Kundrák [13] created a tool life formula based on a rational function with a cubic polynomial as denominator, intending to describe tool life-cutting speed relationship for a wide range of cutting speed values:

$$
T=\frac{C_{T_{1}}}{v_{c}^{3}+C_{T_{2}} v_{c}^{2}+C_{T_{3}} v_{C}}
$$

where $T$ represents tool life, $v_{c}$ represents the cutting speed and $C_{T 1}, C_{T 2}, C_{T 3}$ are constants depending on process conditions. This formula will be employed in the current work for the comparison of predicted and experimental results.

\section{Methodology}

In the present work, an investigation of the applicability of DoE and ANN for tool life prediction of $\mathrm{CBN}$ tools for a wide range of cutting speeds is presented. For all cases, data from [2] are employed to develop ANN models for the prediction of tool life. After some trial runs were performed, the main investigation is conducted; two experimental designs with three factors at four levels, namely Taguchi L16 orthogonal array and $4^{3}$ full factorial design are employed to provide the tool life determination experimental runs, for which values from the actual experiments are used, either directly or indirectly through equation (3). The number of levels was selected to be four, as it can provide the minimum sufficient number of points for the approximation of equation (3), which contains a cubic polynomial. The results from the simulated experiments conducted from these designs are then used to create a predictive model using ANN models. Finally, results from ANN models are 
compared to the results presented in [2] in order to determine at which degree experiments with standard experimental designs can "capture" the tool life curve behaviour for the whole range of cutting speeds.

In this work, the comparison of experimental with ANN predicted curves will be conducted in terms of correlation coefficient R, RMSE, mean absolute error (MAE) and mean percentage error (MPE) in order to assess the level of accuracy of the two approaches. All ANN models employ radial basis functions and are implemented in MATLAB environment with cutting speed $v_{c}(\mathrm{~m} / \mathrm{min})$, feed $f(\mathrm{~mm} / \mathrm{rev})$, depth of cut $a_{p}$ (mm) as inputs and tool life $T(\mathrm{~min})$ as the only output. Spread parameter was determined after a trial-and-error procedure and was kept constant with value $\sigma=0.5$ in every case. The value range for the input variables is $11-120 \mathrm{~mm} / \mathrm{min}$ for the cutting speed, $0.025-0.125$ $\mathrm{mm} / \mathrm{rev}$ for feed and $0.05-0.25 \mathrm{~mm}$ for the depth of cut.

\section{Results and discussion}

The evaluation of results is conducted by comparison of actual values from the experiments, namely 6 cases, presented in [2], which are also displayed in Table 1 . In Table 2 , the performance of each of the two approaches is presented.

Table 1. Characteristics of the cases considered in this study.

\begin{tabular}{|c|c|c||c|c|c|}
\hline No & $\boldsymbol{f}(\mathbf{m m} / \mathbf{r e v})$ & $\boldsymbol{a}_{\boldsymbol{p}} \mathbf{( \mathbf { m m } )}$ & No & $\boldsymbol{f}(\mathbf{m m} / \mathbf{r e v})$ & $\boldsymbol{a}_{p} \mathbf{( \mathbf { m m } )}$ \\
\hline 1 & 0.025 & 0.1 & 4 & 0.050 & 0.05 \\
\hline 2 & 0.075 & 0.1 & 5 & 0.050 & 0.15 \\
\hline 3 & 0.125 & 0.1 & 6 & 0.050 & 0.25 \\
\hline
\end{tabular}

Table 2. Mean values and range of values for R, RMSE, MAE and MPE for the two approaches.

\begin{tabular}{|c|c|c|c|c|}
\hline \multicolumn{5}{|c|}{ Mean values } \\
\hline Approach & R & RMSE & MAE (\%) & MPE (\%) \\
\hline L16+ANN & 0.9714 & 33.3731 & 26.8680 & -21.4911 \\
\hline $4^{3}+$ ANN & 0.9634 & 29.0291 & 23.7730 & 32.2893 \\
\hline \multicolumn{5}{|c|}{ Range of values } \\
\hline Approach & R & RMSE & MAE (\%) & MPE (\%) \\
\hline L16+ANN & $0.9387-0.9827$ & $16.749-53.996$ & $18.909-48.552$ & $-41.513-35.646$ \\
\hline $4^{3}+$ ANN & $0.9187-0.9870$ & $15.919-39.638$ & $19.897-33.230$ & $20.936-58.110$ \\
\hline
\end{tabular}

For the cases where the L16 and ANN approach was used, it is observed that the results are not very satisfactory as error is around $25 \%$; although it is exceeding the widely accepted value of $10 \%$ for prediction errors, the fact that only 16 input/output pairs were used renders this result not totally inacceptable and it will be quite useful for the purposes of the current study. Correlation coefficient value is quite high, indicating that there is strong correlation between predicted and experimentally derived data and RMSE value is not extremely high.

For the cases where the full factorial and ANN approach was used, similar results are generally observed. MAE is below $25 \%$ in all but one case whereas MPE values are quite large in all cases (close to 50\%). Correlation coefficient values are above 0.9 in every case and RMSE mean value of 29.0291 indicates a moderately high degree of error between predicted and experimental curves.

Comparing the results from the two different experimental designs, it can be observed that there are several similarities but also some differences. At first, as can be seen from Table 2, the values of correlation coefficient and errors in each case are close enough, with the second approach exhibiting better mean values for RMSE and MAE, as well as 
narrower value range for these two quantities. These values are indicative of the results portrayed in Figure 1 and Figure 2, as it is evident that none of the two approaches can fully approximate the experimental curve.

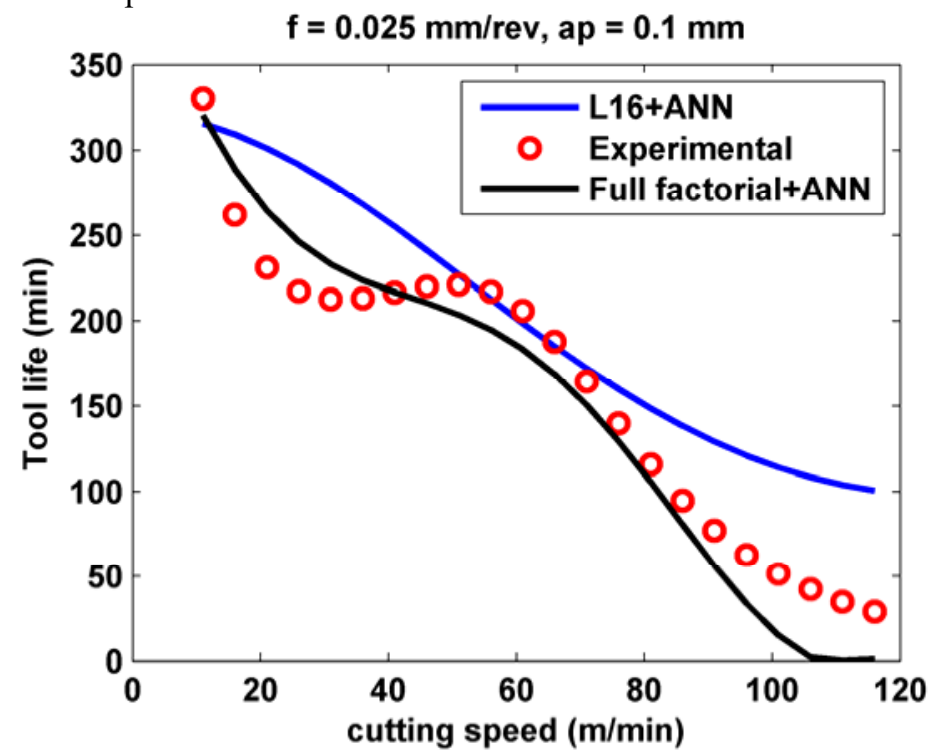

Fig.1. Experimentally and ANN derived curves for $f=0.025 \mathrm{~mm} / \mathrm{rev}, a_{p}=0.1 \mathrm{~mm}$.

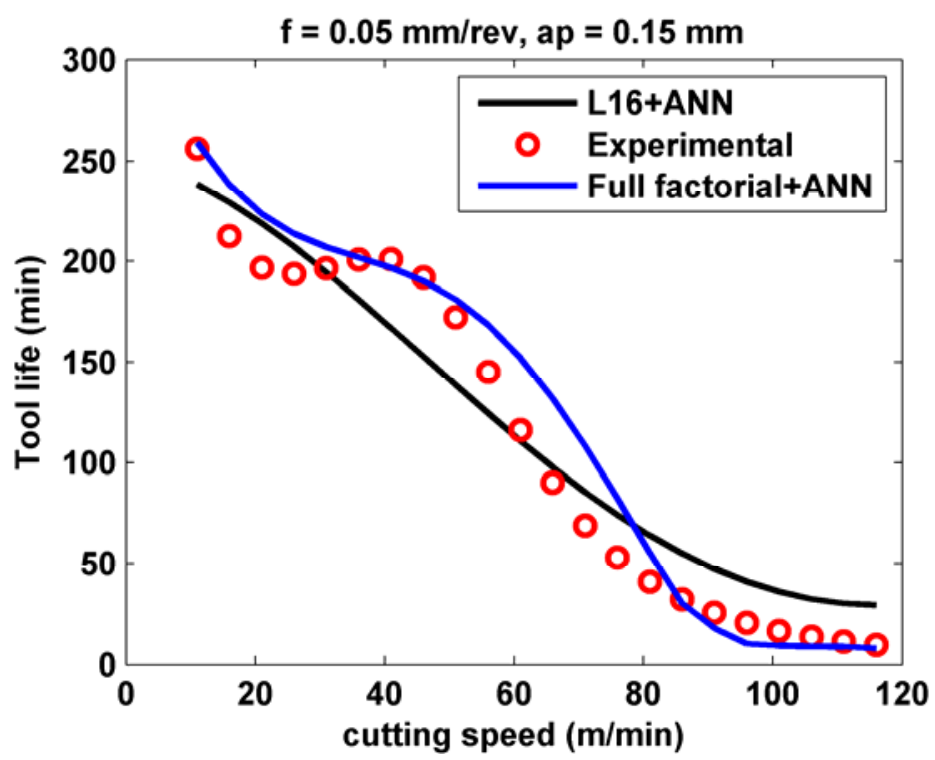

Fig.2. Experimentally and ANN derived curves for $f=0.05 \mathrm{~mm} / \mathrm{rev}, a_{p}=0.15 \mathrm{~mm}$.

It seems that the full factorial approach, which used a lot more data than the L16 approach, i.e. 64 vs. 16, but the same numbers of levels, can provide more accurate estimations of the shape of the experimental curve. Furthermore, the curve derived by the first approach tends to overestimate tool life at low and high speeds, whereas approximates the experimental curve better at moderate cutting speeds. As for the curve derived using the second approach, it can approximate the experimental one adequately at some regions but still the minima and maxima of the experimental curve are not quite accurately predicted. 
Considering that the experimental curve was derived by using 9 levels for the cutting speed instead of 4 , the results are satisfactory for the prediction of tool life but not satisfactory for the prediction of the distinct regions of the tool life-cutting speed curve.

\section{Conclusions}

In this paper, an investigation for the applicability of DoE methods and ANN for the prediction of tool life of CBN cutting tools was conducted. The experiments for the derivation of tool life-cutting speed relationship were conducted using standard experimental designs such as Taguchi method and full factorial method, both at four levels for each factor. Several useful conclusions were drawn:

- Both approaches provided moderate results concerning RMSE, MAE, and MPE, whereas they exhibited high correlation coefficient values. The mean values for MAE and MPE are about $23-32 \%$, which is above the acceptable limit of $10 \%$ but results are not very far from the experimental ones, as indicated by RMSE.

- The ANN trained with results from the full factorial design approach is approximating better the shape of the experimental curve but cannot predict the minima and maxima adequately. It can approximate the experimental curve better at moderate cutting speeds but generally it overestimates the tool life value at the other regions.

- In general, it can be concluded that the non-linear relationship of tool life and cutting speed for a wide range of cutting speeds is difficult to be derived using standard design procedures as it requires data for many cutting speed values to adequately approximate this relationship, something that is not usually the case with DoE methods. However, although the experimental curve was not sufficiently predicted, the deviations of the actual tool life values were not very high. The latter can be useful for preliminary examinations of tool wear and tool life of CBN tools.

In order to develop more accurate models for the approximation of tool life-cutting speed curve, several attempts can be conducted, such as to employ various experimental designs and performance functions for training of the ANN model or a different type of ANN model.

\section{References}

1. A.G. Mamalis, J. Kundrák, M. Horváth, Int J Adv Manuf Tech 20, 475-479 (2002)

2. A.G. Mamalis, J. Kundrák, M. Horváth, J Manuf Sci E-T ASME 127, 328-332 (2005)

3. C. Leone, D. D' Addona, R. Teti, CIRP J Manuf Sci Tech 4, 327-331 (2011)

4. K. Venkata Rao, B.S.N. Murthy, N. Mohan Rao, Measurement 51, 63-70 (2014)

5. D. D’Addona, T. Segreto, A. Simeone, R. Teti, CIRP J Manuf Sci Tech 4, 33-37 (2011)

6. D.M. D’ Addona, R. Teti, Procedia CIRP 12, 252-257 (2013)

7. R.R. Srikant, P.V. Krishna, N.D. Rao, P I Mech Eng B-J Eng 225, 1009-1018 (2011)

8. R. Corne, C. Nath, M. El Mansori, T. Kurfess, Procedia Manuf 5, 1-14 (2016)

9. Q. Liu, Y. Altintas, Int J Mach Tool Manu, 39, 1945-1959 (1999)

10. P. Krishnakumar, K. Rameshkumar, K.I. Ramachandran, Procedia Comp Sci 50, 270275 (2015)

11. S.S. Gill, R. Singh, J. Singh, H. Singh, Expert Syst Appl 39, 4171-4180 (2012)

12. A.I. Azmi, Adv Eng Softw 82, 53-64 (2015)

13. J. Kundrák, The scientific principles of increasing the effectiveness of inner surfaces cutting with CBN tools, (Kharkov, 1996) 